\title{
A fuzzy definition of the spatial relation "surround" - Application to complex shapes
}

\author{
Maria Carolina Vanegas ${ }^{1,2}$ Isabelle Bloch $^{1}$ Jordi Inglada $^{2,3}$ \\ ${ }^{1}$ Institut Telecom, Télécom ParisTech, CNRS-LTCI UMR 5141, Paris, France \\ ${ }^{2}$ CNES, Toulouse, France \\ ${ }^{3}$ CESBIO UMR 5126, Toulouse, France
}

\begin{abstract}
Spatial relations are of great importance for image understanding. In this paper we focus on the "surround" relation. We propose to represent the relation as a fuzzy set in the spatial domain, which considers the imprecision of the relation itself, the morphology of the reference object, and the distance to the target object. This fuzzy set represents the region which surrounds some reference objects. The degree to which another object satisfies this relation with respect to the reference object can then be derived. The definition is illustrated on real objects with complex shapes from satellite images.
\end{abstract}

Keywords: fuzzy spatial relations, surround.

\section{Introduction}

Spatial relationships encode important structural information and have shown to be useful in object recognition and image interpretation tasks. The aim of this paper is to propose a definition for the spatial relation "surround". This paper is motivated by the importance of the "surround" relation in remote sensing images, where for instance, we have structures on the sea (boats, harbors) which are surrounded by it. For such complex objects, existing definitions are not sufficient. Here we propose new definitions that fit the intuition, and take into account the shape of the objects as well as their distance.

We say that the relation " $A$ is surrounded by $B$ " is satisfied if $A$ is able to see $B$ in almost all the directions. The notion of $B$ being "in almost all" directions with respect to $A$ is by definition imprecise. It is imprecise in the sense that "almost all" is not a well defined quantity. Moreover as highlighted in $[1,2]$, the notion of being "in a direction $\alpha$ " from another object is also imprecise. Thus, the fuzzy set theory is appropriate for modeling this relation.

When modeling a fuzzy spatial relation two questions are raised [3]:

(i) to which degree is the relation satisfied between two objects?

(ii) which is the region of space where the relation to a reference object is satisfied (to some degree)?
The manner in which the relation is modeled depends on the question to be answered. To our knowledge, most of the models which have been proposed for the "surround" relation focus on answering the first question. These models are discussed in Section 2. In this work we propose a new model for the "surround" relation which focuses on answering the second question. There are several advantages of using a model that answers (ii) rather than one which answers (i). For instance, if we need to find the target objects which satisfy the relation with respect to a reference object $B$, then we compute the region once, and observe which are the objects which are compatible with this region. While if we use a model which answers (i), then it is necessary to compute the relation between $B$ and every object in the image, which can have a higher computational cost. Another advantage is that it is possible to combine several spatial relations, which are represented in the same manner by fusioning their respective regions, as in [4].

In Section 2 we review some of the definitions proposed in the literature for the "surround" relation. Then, inspired by some of these works, we propose a novel definition of fuzzy surround in Section 3. The contributions are twofold: first we propose a definition as a spatial fuzzy set (a fuzzy landscape), which defines the degree of satisfaction of the relation to a reference object for each point of the space; secondly, we propose to include several important pieces of information in the definition, such as the potential imprecision of the objects (i.e. dealing with fuzzy sets), the distance to the target object, and shape information. In Section 4 an example with a complex shaped reference object illustrates that the proposed definition provides adequate results, in accordance with intuition.

\section{Related work}

Three types of definitions for the relation " $A$ is surrounded by $B$ " have been proposed in the literature. The relation can be first defined as the conjunction of the degrees of primitive directional relations $[2,5]$. For instance, in the $2 \mathrm{D}$ case, the degree to which " $A$ surrounds by $B$ " is equal to the conjunction of the degrees of satisfaction of the relations "left of", "right of", "above" and "below" when 
$B$ is the reference object and $A$ is the target object. This definition is very strict since it requires that the target object is in "all" the directions with respect to the reference object. A more flexible relation, accounting for partial surrounding situations, may be better suited. The second type is the topological surround of [6] which depends on the angular change of the paths going from the points on the boundary of $A$ to the outside of the image (or spatial domain) in order to not intersect $B$. This definition corresponds to a crisp notion which is again not flexible enough. Figure 1(a) shows a situation for which a point is not topologically surrounded by an object since there is a straight path (shown in red) from the point to the outside of the image which does not intersect $B$. Nonetheless we would like to have a degree of satisfaction of the "surround" relation which is greater than zero because $p$ is able to see $B$ in several directions.

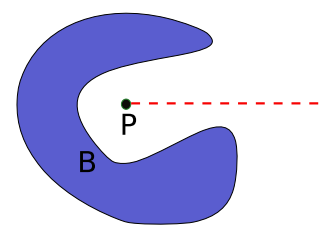

(a)

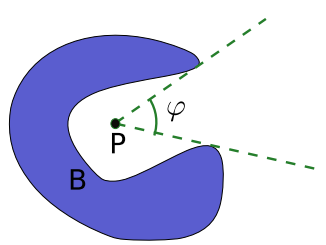

(b)
Figure 1: (a) In red a direct path from the point to the outside of the image is shown, demonstrating that the topological surroundedness is not satisfied. (b) In green the angular interval $\varphi$ for which $r_{\theta}(p, B)=0$. The visual surroundedness has a degree of satisfaction less than 1 since $\varphi>0$.

Finally, the third type deals with the angular coverage. It is the closest to what we propose in this paper. In the following we detail the different approaches used in this type of definitions.

In [6] Rosenfeld et al. define the visual surroundedness, first for a point $p$ "surrounded by" an object $B$, and then for any two objects. Let $p \in \mathcal{S}$ be a point of the spatial domain $\mathcal{S}$ and $B$ an object $B \subseteq \mathcal{S}$. Rosenfeld et al. first introduce the function $r_{\theta}$ :

$$
r_{\theta}(p, B)= \begin{cases}1 & \text { if } \exists b \in B \text { such that } \angle\left(\overrightarrow{p b}, \vec{u}_{x}\right)=\theta \\ 0 & \text { otherwise. }\end{cases}
$$

where $\vec{u}_{x}$ is the unitary vector in the direction of the $x$-axis, and $\angle\left(\vec{p} \vec{b}, \vec{u}_{x}\right)$ denotes the angle between the segment $[p, b]$ and the $x$-axis. The value of $r_{\theta}(p, B)$ is equal to one, if there exists a ray from $p$ in the direction $\theta$ which intersects $B$. Then the degree to which a point $p$ is "surrounded by" an object $B$ is given by:

$$
\mu_{\text {visual_surround }}(p, B)=\frac{1}{2 \pi} \int_{0}^{2 \pi} r_{\theta}(p, B) d \theta
$$

This degree measures the portion of angular coverage of $p$ by $B$. In Figure 1(b) the degree of satis- faction is $\mu_{\text {visual_surround }}(p, B)=1-\frac{\varphi}{2 \pi}$, where $\varphi$ is equal to the angular interval for which $r_{\theta}(p, B)$ is equal to zero. The extension to see whether an object $A$ is surrounded by an object $B$ is straightforward:

$\mu_{\text {visual_surround }}(A, B)=\min _{p \in \partial A} \mu_{\text {visual_surrounds }}(p, B)$

The definition of visual surroundedness is in agreement with the relation we want to define. If an object $A$ sees $B$ in "almost all" directions, then there is a large angular coverage of $A$ by $B$ and therefore $\mu_{\text {visual_surround }}(A, B)$ is high.

Another definition of "surround" was proposed in [7]. To evaluate the degree to which an object $A$ is surrounded by $B$ the maximum angle $\varphi$ between two tangent lines of $B$ passing through a point of $A$ is computed (this angle is the same as the one illustrated in green in Figure 1(b)). The degree of satisfaction of the relation decreases as $\varphi$ gets larger. So the membership function defining the surround relation is expressed by:

$$
\mu_{\text {surrounds }}(\varphi)= \begin{cases}\cos ^{2}\left(\frac{\varphi}{2}\right) & \text { if } 0 \leq \varphi \leq \pi \\ 0 & \text { otherwise }\end{cases}
$$

Then the degree to which a region $B$ surrounds a region $A$ is obtained by the center of gravity of the compatibility distribution of the angle histogram $H^{B}(A)$ [7] between $A$ and $B$ and $\mu_{\text {surrounds }}(\varphi)$. This definition is also in agreement with the definition of surroundedness. A high satisfaction value is obtained for the situation shown in Figure 1(b).

A definition based on angle calculation is presented in [8]. The normalized angle or force histogram $H^{A}(B)$ is computed. Then for every $\alpha$ cut $H^{A}(B)_{\alpha}$ of $H^{A}(B)$ the angle $z_{1}$ is defined as the largest angle interval which is not included in $H^{A}(B)_{\alpha}$. The degree of surround for an $\alpha$-cut is given by a decreasing function of $z_{1}$, and in [8] the function $\max \left(0,1-\frac{z_{1}}{\pi}\right)$ is used. Finally, the degree of surround is given by an integration over all the $\alpha$-cuts. This approach does not measure the angle coverage, but it only observes the largest angular interval which is not covered. For instance, if $B$ is a disconnected object composed of three points which are arranged to form an equilateral triangle, and $A$ is just a point located in the center of the triangle, then for every $\alpha$-cut the angle $z_{1}$ is equal to $\frac{\pi}{3}$ and the degree of surround is 0.67 . This degree is not in accordance with the definition of surroundedness that we want to define, since $B$ only sees $A$ in very few directions.

The surround relations proposed in $[6,7]$ are in agreement with the relation we want to define. These definitions allow assessing the degree to which the relation is satisfied between two objects (i.e. answering the first question raised in introduction). In the following section we concentrate on defining a region of space, also known as fuzzy landscape, 
where the relation is satisfied, thus answering the second question. Moreover, in order to deal with complex shapes, we propose as an additional contribution to include information on the morphology of the objects as well as their distance to the target objects, which were not directly taken into account in existing definitions.

\section{Definition of surroundedness as a fuzzy landscape}

We first define the relation for a crisp reference object, and then we extend it to the case of fuzzy objects.

\subsection{Crisp reference object}

Using the same idea as the visual surroundedness of [6], we define the angular coverage of a point by a region $B$. For every point $p \notin B$ the angular coverage is equal to the total angular length of the angular intervals for which $p$ is able to see $B$, that is:

$$
\theta_{\text {coverage }}(B)(p)=\int_{0}^{2 \pi} r_{\theta}(p, B) d \theta
$$

The region representing "surrounded by $B$ " can then be defined as a fuzzy region of space whose membership function is expressed as:

$$
\mu_{\text {surround }}(B)(p)=f\left(\theta_{\text {coverage }}(B)(p)\right)
$$

where $f:[0,2 \pi] \rightarrow[0,1], f(0)=0$ and $f(2 \pi)=$ 1. The purpose of $f$ is to define the membership function defining the semantics of "almost all" for the angular coverage.

Figure 2 shows some examples of this definition of surroundedness applied to synthetic objects. In these examples $f$ was defined as:

$$
f(x)= \begin{cases}1 & \text { if } x \geq \frac{5 \pi}{4} \\ \frac{x-\frac{\pi}{2}}{\frac{3 \pi}{4}} & \text { if } \frac{\pi}{2} \leq x<\frac{5 \pi}{4} \\ 0 & \text { if } x<\frac{\pi}{2}\end{cases}
$$

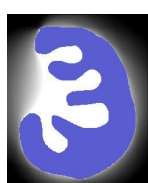

(a)

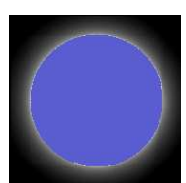

(b)

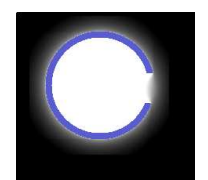

(c)

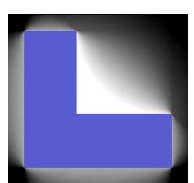

(d)
Figure 2: Fuzzy landscapes obtained with the definition of Equation 6 when the blue object is used as reference object. The brightest grey level corresponds to the higher membership values. We note grey levels "around" the objects which do not correspond to the wanted property "to be surrounded by" the object.

The results shown in Figure 2 are not in complete agreement with the notion of angular coverage. Some non zero membership areas appear around convex parts of objects, which is counter-intuitive (see e.g. the convex object in Figure 2(b)), since a convex object cannot surround another object.

On the other hand, one can interpret the surround relation by considering that $A$ is "surrounded by" $B$, if there is a portion of the boundary of $B$ that goes around $A$. In [9] the linguistic aspects of the relation "go around" are analyzed, which leads to consider that a path $C$ "goes around" an object $A$ if $A$ and $C$ do not intersect, and if $A$ intersects the convex hull of $C$. Consequently, the "surround" relation should only take place when the reference object has concavities. Moreover, the portion of boundary belonging to the concavities is the one which "goes around" the other object. As a result, the angular coverage should be computed only using the points on the boundary of $B$ which lie in a concavity. A point lies in a concavity if it is in $\partial B \backslash \partial C H(B)$, where $C H(B)$ and $\partial B$ are the convex hull and boundary of $B$, respectively. A point $b$ that lies in a concavity sees $p$ if the segment $[p, b[$ does not intersect $B$. Thus, to take into account the concavities as well as the notion of visibility, we redefine the function $r_{\theta}(p, B)$ of [6] (Equation 1). We only consider the segments which have an endpoint in $\partial B \backslash \partial C H(B)$ and do not intersect $B$, using the same idea as for the admissible segments of [6]:

$$
\tilde{r}_{\theta}(p, B)=\left\{\begin{array}{cc}
1 \quad \text { if } \exists b \in \partial B \backslash \partial C H(B) \text { such that } \\
\quad \angle\left(\overrightarrow{p b}, \vec{u}_{x}\right)=\theta \text { and }[p, b[\cap B=\emptyset \\
0 \quad \text { otherwise. }
\end{array}\right.
$$

Figure 3 shows an example of the angular interval where $r_{\theta}(p, B)=0$ and $\tilde{r}_{\theta}(p, B)=0$, labeled as $\varphi$ and $\tilde{\varphi}$, respectively. We can see that $\varphi<\tilde{\varphi}$, and therefore it is necessary to add the notion of visibility to penalize the points which are not really seen by a concavity.

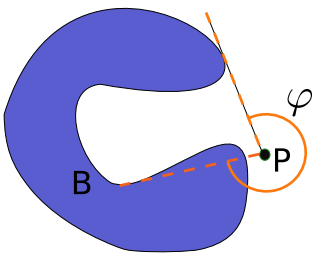

(a)

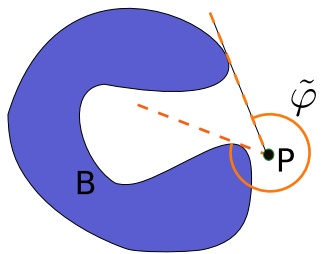

(b)
Figure 3: In orange the angular interval $\varphi$ and $\tilde{\varphi}$ for which $r_{\theta}(p, B)=0$ in (a) and $\tilde{r}_{\theta}(p, B)=0$ in (b).

As a result, the angular coverage should be computed using $\tilde{r}_{\theta}$ :

$$
\theta_{\text {coverage_CH }}(B)(p)=\int_{0}^{2 \pi} \tilde{r}_{\theta}(p, B) d \theta
$$

Again the fuzzy landscape representing the region surrounded by $B$ is obtained by applying $f\left(\theta_{\text {coverage_CH}}\right)$ to each point in $\mathcal{S}$, where $f$ has the same properties as the function presented in Equa- 
tion 6:

$$
\mu_{\text {surround }}(B)(p)=f\left(\theta_{\text {coverage_CH }}(B)(p)\right)
$$

Figure 4 shows the fuzzy landscapes for the reference objects in Figure 2. These fuzzy landscapes have high membership values for the points which are in the interior of the concavities of the reference object and lower ones for the points which are away from the concavities. Clearly, there is no region of space which satisfies the surrounded relation when the reference object is convex (Figure 4(b)).

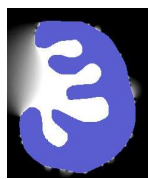

(a)

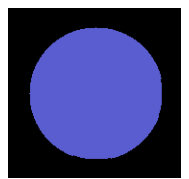

(b)

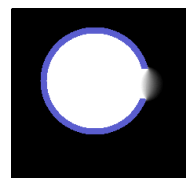

(c)

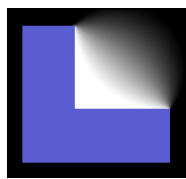

(d)
Figure 4: Fuzzy landscapes obtained with Equation 10. The defect presented in Figure 2 no longer exists.

\subsection{Evaluation of the relation between two objects}

The proposed method allows answering the second type of question. Answering the first type of question can then be derived from the fuzzy landscape. The evaluation of to which degree an object " $A$ is surrounded by $B$ " is done by comparing how well $A$ matches the region having a high membership value in the fuzzy landscape, as in [5]. If $A$ is a fuzzy object, defined through a membership function $\mu_{A}$ over $\mathcal{S}$, we can use a comparison measure between fuzzy sets, see [10] for a review of these measures. For instance, we can use a necessity $(N) /$ possibility (П) interval [11], where $N$ is defined as an inclusion degree using a t-conorm $T$ :

$$
N\left(A, \mu_{\text {surround }}(B)\right)=\inf _{p \in \mathcal{S}} T\left(\mu_{\text {surround }}(B)(p), 1-\mu_{A}(p)\right)
$$

and $\Pi$ is defined as an intersection degree through a t-norm $t$ :

$$
\Pi\left(A, \mu_{\text {surround }}(B)\right)=\sup _{p \in \mathcal{S}} t\left(\mu_{\text {surround }}(B)(p), \mu_{A}(p)\right)
$$

The degree $N$ measures the degree of inclusion of $A$ in $\mu_{\text {surround }}(B)$ and represents a pessimistic evaluation. The degree $\Pi$ represents an optimistic evaluation and measures the degree of intersection between $A$ and $\mu_{\text {surround }}(B)$. Another possible measure [10] can be a satisfiability measure such as a normalized intersection:

$M\left(A, \mu_{\text {surround }}(B)\right)=\frac{\sum_{p \in \mathcal{S}} t\left(\mu_{\text {surround }}(B)(p), \mu_{A}(p)\right)}{\sum_{p \in \mathcal{S}} \mu_{A}(p)}$

\subsection{Considering the distance of target object}

The fuzzy landscape defined by Equation 10 is adequate when the size of the target object is comparable to the size of the concavity. However, when this is not the case, the obtained fuzzy landscape is not appropriate. For instance, if $B$ is the reference object in Figure $5(\mathrm{a}), A_{1}$ should have a high satisfaction degree for the relation surrounded, since there is a portion of the boundary of $\partial B \backslash \partial C H(B)$ which goes around the object and which is close to it. On the contrary, intuitively, the object $A_{2}$ should not have a high satisfaction degree of the relation surround. Even if there is a portion of the boundary of $\partial B \backslash \partial C H(B)$ which goes around the object, some of the points belonging to this portion of boundary are so "far" from $A_{2}$, that they are not considered visible from $A_{2}$. The interpretation of "far" and "near" according to an object can depend on its intrinsic characteristic, for instance on its size [12].

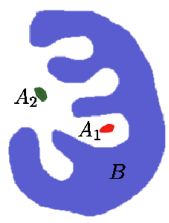

(a)

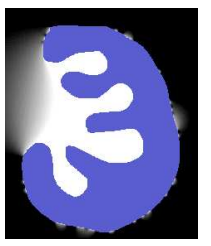

(b)

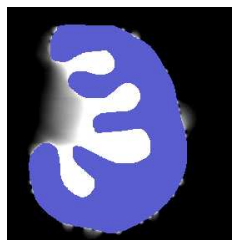

(c)
Figure 5: Illustration of the fuzzy landscapes when not considering the distance of the target object and when considering it. (a) The reference object is denoted by $B$, and two target objects by $A_{1}$ and $A_{2}$. (b) $\mu_{\text {surround }}(B)$ using Equation 10. (c) $\mu_{\text {surround }}\left(B, \mu_{n}\right)$ using Equation 16.

The second and third columns of Table 1 show the results of evaluating the satisfaction of the relation "surround by" using Equation 10. Both the $[N, \Pi]$ and the $M$ measures give similar results for $A_{2}$ and $A_{1}$, although, intuitively, $A_{1}$ should better satisfy the relation since it is more inside the concavity of $B$ (hence more surrounded).

\begin{tabular}{|c||c|c||c|c|}
\hline \multirow{2}{*}{$\begin{array}{c}\text { Target } \\
\text { object }\end{array}$} & \multicolumn{2}{c||}{$\begin{array}{c}\mu_{\text {surround }}(B) \\
\text { Equation } \\
\end{array}$} & \multicolumn{1}{c||}{10} & \multicolumn{2}{c|}{$\begin{array}{c}\mu_{\text {surround }}\left(B, \mu_{n}\right) \\
\text { Equation } \\
16\end{array}$} \\
\cline { 2 - 5 } & {$[N, \Pi]$} & $M$ & {$[N, \Pi]$} & $M$ \\
\hline \hline $\mathrm{A} 1$ & {$[1.0,1.0]$} & 1.0 & {$[1.0,1.0]$} & 1.0 \\
$\mathrm{~A} 2$ & {$[0.73,1.0]$} & 0.85 & {$[0.21,0.64]$} & 0.48 \\
\hline
\end{tabular}

Table 1: Evaluation of the surround relation using Equations 10 and 16, for objects in Figure 5(a).

In order to include the "near" relation of the target object with respect to the reference object, the measure of angular coverage should be modified.

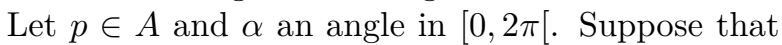
there exists $q \in \partial B \backslash \partial C H(B)$ which intersects the ray emanating from $p$ in the direction $\alpha$. However, if $q$ is not "near" $p$ according to $A$, then it should be 
considered as if there was no point in $\partial B \backslash \partial C H(B)$ in the direction $\alpha$.

Let $\mu_{n}$ be a function over $\mathbb{R}^{+}$which represents the "near" distance according to the target object. It can defined as a trapezoid function, where the parameters are adjusted according to the size of the target object. If we want to evaluate the relation for several target objects of comparable size, then we can define $\mu_{n}$ according to this size. As a result, we define the function $\tilde{r}_{\theta}\left(p, B, \mu_{n}\right)$ as:

$\tilde{r}_{\theta}\left(p, B, \mu_{n}\right)= \begin{cases}\mu_{n}\left(d_{E}(p, q)\right) & \text { if } \exists q \in \partial B \backslash \partial C H(B) \\ & \text { such that } \angle\left(\overrightarrow{p q}, \vec{u}_{x}\right)=\theta \\ & \text { and }[p, q[\cap B=\emptyset, \\ 0 & \text { otherwise. }\end{cases}$

The function $\tilde{r}_{\theta}\left(p, B, \mu_{n}\right)$ is equal to the membership value of the "near" relation of the point in $\partial B \backslash$ $\partial C H(B)$ which lies on the ray emanating from $p$ in the direction $\theta$. The point in $\partial B \backslash \partial C H(B)$, which satisfies this, is uniquely defined. Using $\tilde{r}_{\theta}\left(p, B, \mu_{n}\right)$ we can define the angular coverage which takes into account the size of the desired target object or objects, as well as considering the points of the convex hull of the reference object $B$ :

$$
\theta_{\text {coverage_CH }}\left(B, \mu_{n}\right)(p)=\int_{0}^{2 \pi} \tilde{r}_{\theta}\left(p, B, \mu_{n}\right) d \theta \text {. }
$$

Finally, the degree of surround is given by:

$$
\mu_{\text {surround }}\left(B, \mu_{n}\right)(p)=f\left(\theta_{\text {coverage_CH}}\left(B, \mu_{n}\right)(p)\right) \text {. }
$$

Figure 5(c) shows the fuzzy landscape defined by Equation 16. For this experiment we modeled $\mu_{n}$ as a trapezoid function:

$$
\mu_{n}(x)= \begin{cases}1 & \text { if } x \leq d_{1} \\ \frac{d_{2}-x}{d_{2}-d_{1}} & \text { if } d_{1}<x \leq d_{2} \\ 0 & \text { otherwise }\end{cases}
$$

The parameters $d_{1}$ and $d_{2}$ are associated with the imprecision of defining the relation "near". For instance we can use a very restrictive function were:

$$
\begin{aligned}
& d_{1}=4 l_{\text {average }} \\
& d_{2}=5 l_{\text {average }}
\end{aligned}
$$

where $l_{\text {average }}$ is equal to the average of the lengths of the maximum diameter. In this particular case, we have use $l_{\text {average }}=25.3$, and so $d_{1}=101.2$ and $d_{2}=126.5$. We have chosen these parameters just to illustrate the influence of considering the distance when evaluating the "surround" relation. However, for a real application these parameters can be learned. The fuzzy landscape of Figure 5(c) has high membership values in the regions where the concavities are small, and can go around an object of the same size as $A_{1}$ or $A_{2}$. The last two columns of Table 1 show the results of evaluating the satisfaction of the relation "surrounded by" defined by Equation 16. Both the mean and the necessity/possibility measures give results which fit with the intuition, indicating that $A_{1}$ completely satisfies the relation, while $A_{2}$ has a much lower satisfaction, as expected.

Attaching the fuzzy landscape of surround to the characteristics of the target object, such as size, might seem restrictive, since one of the advantages of computing a fuzzy landscape is that one has to compute the landscape only once, and then it can be used to evaluate the relation for several objects. However, other advantages of the fuzzy landscape, such as determining the region of space where it is possible to find a particular target object that satisfies the relation, continue to be valid. Other applications, such as evaluating the relation for several objects of similar size can also be envisaged.

\subsection{Extension to fuzzy objects}

When $B$ is a fuzzy object with membership $\mu_{B}$, the function $\tilde{r}_{\theta}\left(p, B, \mu_{n}\right)$ and the notion of angular coverage should be adapted. When $B$ is a fuzzy set, its convex hull is also a fuzzy set. The $\alpha$-cuts of a fuzzy set $\mu$ are nested, as well as their convex hulls. So, the convex hull of a fuzzy set can be defined as the convex hull of its $\alpha$-cuts [13]:

$$
\left(C H\left(\mu_{B}\right)\right)_{\alpha}=C H\left(\left(\mu_{B}\right)_{\alpha}\right) .
$$

Then the notion of angular coverage $\theta_{\text {coverage_CH}}\left(B, \mu_{n}\right)(p)$ is directly extended by replacing $\partial B \backslash \partial C H(B)$ by the fuzzy set $\mu_{\partial B \backslash \partial C H(B)}$ defined as:

$$
\mu_{\partial B \backslash \partial C H(B)}(p)=t\left[\mu_{\partial B}(p), c\left(\mu_{\partial C H(B)}(p)\right)\right],
$$

where $c$ is a fuzzy complementation, $t$ a t-norm and $\mu_{\partial B}$ represents the fuzzy boundary of $\mu_{B}$, which can be computed as in [14] by using mathematical morphology. For instance, the membership function of the internal boundary of a fuzzy set $\mu_{X}$ is defined as:

$$
\mu_{\partial X}(p)=t\left[D_{V_{c}}\left(c\left(\mu_{X}\right)\right)(p), \mu_{X}(x)\right]
$$

where $V_{c}$ is a fuzzy structuring element representing the notion of neighborhood between two points.

Now the function $\tilde{r}_{\theta}\left(p, B, \mu_{n}\right)$ is adapted to the case of a fuzzy object $B$ as follows:

$$
\begin{gathered}
\max _{\theta}\left(p, B, \mu_{n}\right)= \\
\max _{\left\{q \in \mathcal{S} \mid \angle\left(\overrightarrow{p q}, \vec{u}_{x}\right)=\theta\right\}} t\left[\mu_{n}\left(d_{E}(p, q)\right), \mu_{\partial B \backslash \partial C H(B)}(q),\right. \\
\mu_{\text {inclusion }}\left(\left[p, q\left[, B^{c}\right)\right],\right.
\end{gathered}
$$

where $t$ is a t-norm, $\mu_{\text {inclusion }}$ corresponds to the degree of inclusion (for instance the one used in Equation 11). For every $q \in \mathcal{S}$, for which the segment $[p, q]$ makes an angle $\theta$ with the horizontal axis, we take the value which better satisfies conjunctively the three conditions used for the definition of this 
function in the crisp case. The first condition refers to the fact that $p$ and $q$ should be "near" according to the function $\mu_{n}$. The second one establishes that $q$ should belong to $B$ and finally the third one states that the segment $[p, q$ [ should be included in the fuzzy complement of $B$. When $B$ is crisp, Equations 23 and 14 give the same result.

To extend the definition in Equation 10, where the distance to the target object is not taken into account, the function $\tilde{r}_{\theta}(p, B)$ is changed to:

$$
\begin{aligned}
& \tilde{r}_{\theta}(p, B)= \\
& \max _{\left\{q \in \mathcal{S} \mid \angle\left(\overrightarrow{p q}, \vec{u}_{x}\right)=\theta\right\}} t\left[\mu_{\partial B \backslash \partial C H(B)}(q), \mu_{i n c}\left(\left[p, q\left[, B^{c}\right)\right] .\right.\right.
\end{aligned}
$$

As for the aforementioned case, this function represents the conjunction of the two conditions which define $\tilde{r}_{\theta}(p, B)$ in the crisp case.

\subsection{Properties}

The fuzzy landscape obtained by the definition of surround that does not take into account the distance to the object (Equation 10) is always larger than the one obtained with the definition that takes into account the distance to the object (Equation 16). More specifically, for every $x \in \mathcal{S}$ and $\mu_{n}$, we have $\mu_{\text {surround }}(B)(x) \geq \mu_{\text {surround }}\left(B, \mu_{n}\right)(x)$. Moreover, the equality is obtained when $\mu_{n}$ is the constant function equal to 1 . Therefore, the definition of surround of Equation 10 is a particular case of the definition of Equation 16. Therefore all the properties that are satisfied by $\mu_{\text {surround }}\left(B, \mu_{n}\right)$, are also satisfied by $\mu_{\text {surround }}(B)$.

The proposed surround relation is invariant with respect to rigid geometrical transformations (translation and rotation). It is invariant with respect to scale if and only if $\mu_{n}$ is invariant with respect to scale.

The definition of surround is increasing with respect to $\mu_{n}$. For every $\mu_{n 1}, \mu_{n 2}$ such that $\mu_{n 1}(x) \leq$ $\mu_{n 2}(x)$ for every $x \in \mathcal{S}$, then $\mu_{\text {surround }}\left(B, \mu_{n 1}\right)(x) \leq$ $\mu_{\text {surround }}\left(B, \mu_{n 2}\right)(x)$.

\subsection{Computational complexity}

Assume that the image is a square with sides equal to $\sqrt{N}$ and $N$ the number of points. Let $N_{B}$ be the number of points in the reference object and $N_{d}$ the length of the core of $\mu_{n}$. The complexity of computing the convex hull is $O\left(N_{B} \log N_{B}\right)^{1}$. The complexity of computing the function $\tilde{r}_{\theta}(p, B)$ of Equation 9 is of the order of $N^{2}$. Therefore the complexity of computing $\mu_{\text {surround }}(B)$ defined by Equation 10 is $O\left(N_{B} \log N_{B}+N^{2}\right)$.

The complexity of computing $\mu_{\text {surround }}\left(B, \mu_{n}\right)$ defined by Equation 16 is $O\left(N_{B} \log N_{B}+N_{d} N\right)$, since the complexity of computing the function $\tilde{r}_{\theta}\left(p, B, \mu_{n}\right)$ is of the order of $N_{d} N$.

\footnotetext{
${ }^{1}$ We assume that the $\mathrm{q}$-hull algorithm is used for computing the convex hull [15].
}

\section{Illustrative example}

In this section we present an example to illustrate the defined relation. We consider an example in satellite images, and objects with complex shapes. As a reference object, we consider the sea, which exhibits several concavities, shown in Figure 6(b). This reference object is a fuzzy region, where the white pixels represent the points of space with a high membership value, while the black ones represent the points with low membership value. The target objects are the boats shown in Figure 6(c). The sizes of the target objects are comparable, therefore the relation can be evaluated by using the same fuzzy landscape for all objects.

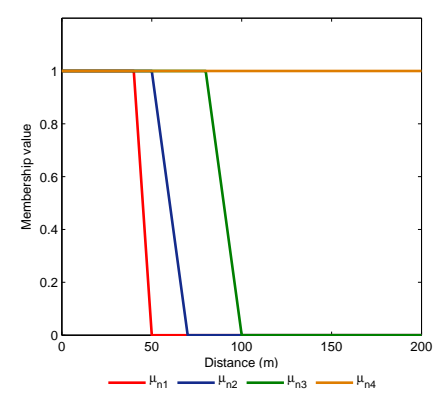

Figure 7: Membership functions for $\mu_{n}$.

To observe the influence of the distance relation involved in the computation of $\mu_{\text {surround }}$, we used different membership functions to represent the "near" notion, as illustrated in Figure 7. There are four membership functions representing different degrees of permissiveness of "near". The function $\mu_{n 1}$ is very strict. The functions $\mu_{n 2}$ and $\mu_{n 3}$ are more in accordance with the size of the target objects, since the average lengths of the object's main directions is $l_{\text {average }}=10.3$. Finally, $\mu_{n 4}$ is a constant membership function equal to one, which gives a fuzzy landscape equivalent to the one in Equation 10.

The corresponding fuzzy landscapes are shown in Figure 8 and the evaluation for the target objects is shown in Table 2. The fuzzy landscape of Figure 8(a) is very restrictive, and it is not well adapted to evaluate the relation for the selected target objects. It represents a situation where the target objects should be very small, and the reference object must be almost touching them. This fuzzy landscape does not allow us to cope with the imprecision linked to the segmentation of the reference object. The restrictions imposed by the function "near" are not appropriate for the situation. For instance, in the evaluation of the yellow boat (6), the large interval $[N, \Pi]$ exhibits an ignorance with respect to the satisfaction of the relation. Nonetheless, there is no ambiguity that this boat is surrounded by the sea.

The second and third landscapes (Figures 8(b) 


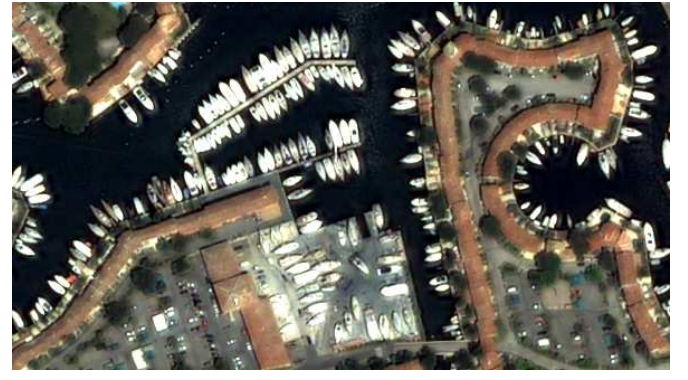

(a)

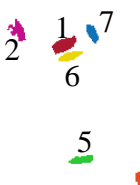

13

(c)

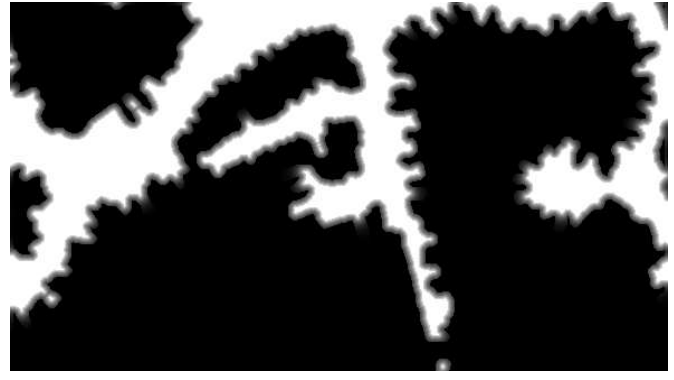

(b)

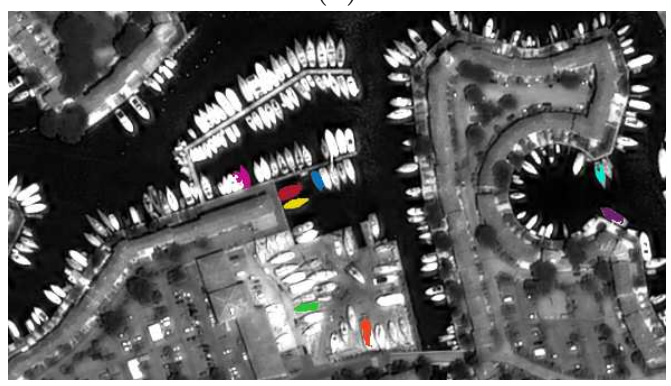

(d)

Figure 6: Reference and target objects used to evaluate the relation surround. (a) Original image. (b) Reference object: the sea (white region). (c) Target objects. (d) Target objects superimposed on the original image.

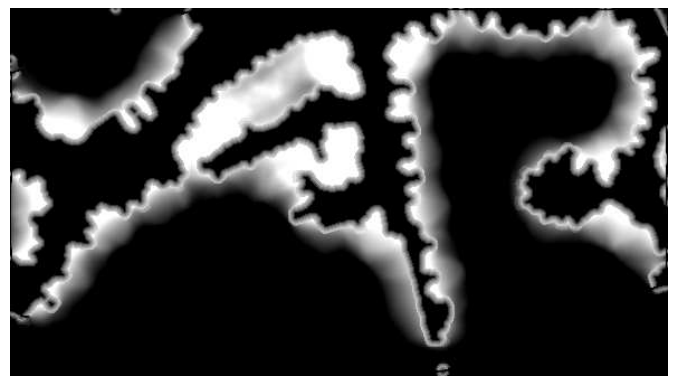

(a)

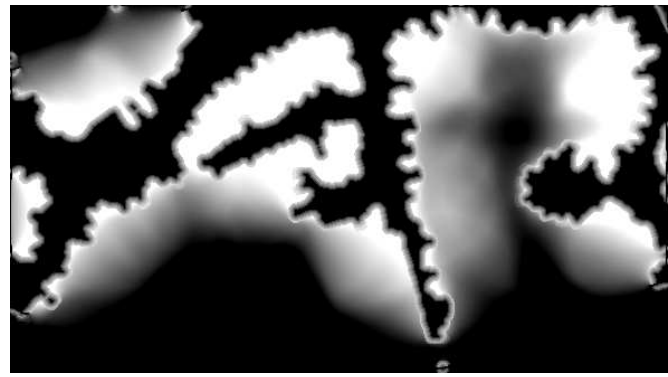

(c)

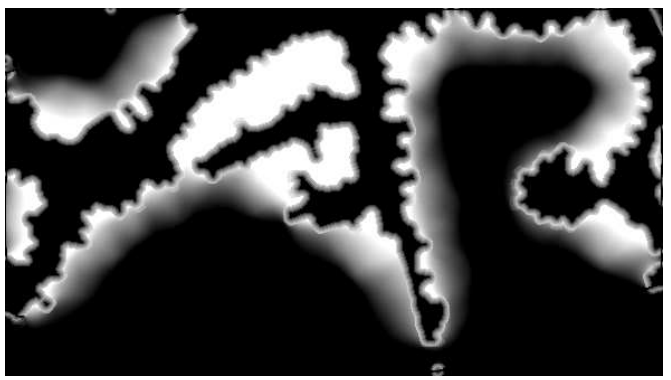

(b)

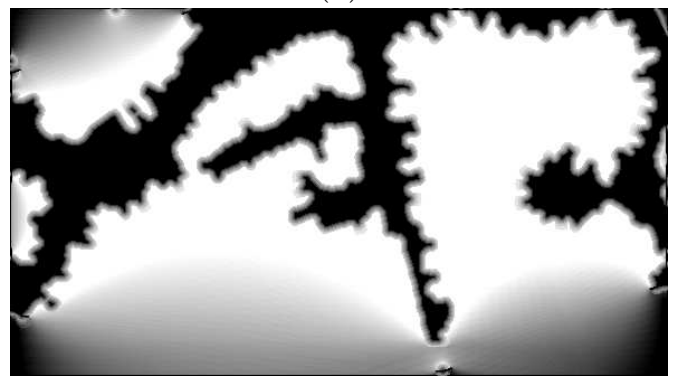

(d)

Figure 8: Fuzzy landscapes representing the relation surround for different distance membership functions shown in Figure 7. (a) Using $\mu_{n 1}$. (b) Using $\mu_{n 2}$. (c) Using $\mu_{n 3}$. (d) Using $\mu_{n 4}$.

and $8(\mathrm{c})$ ) are more suitable for evaluating the relation. We obtain high satisfaction degrees for all the boats, except the green (5) and the orange (3) ones, as expected. The satisfaction values obtained for this relation are in better accordance with the intuition. The intervals $[N, \Pi]$ are shorter, showing less ambiguity in the satisfaction of the relation for these target objects. Moreover, the fuzzy landscape of Figure 8(b) corresponds to $\mu_{n}$ obtained using the parameters of Equations 18 and 19. This suggests that these parameters are an appropriate choice.

The last fuzzy landscape (Figure $8(d)$ ) is very permissive. According to the first property listed in Section 3.5, this fuzzy landscape corresponds to the case where the size of the targets is not taken into account. The results obtained for this situation are not really significant, since a high satisfaction degree $M$ is obtained for all the target objects, while, intuitively, the green (5) and orange (3) boats should have a low satisfaction value. 


\begin{tabular}{|l||c|c||c|c||c|c||c|c||}
\hline \multirow{2}{*}{$\begin{array}{l}\text { Target Objects } \\
\text { Figure 6(c) }\end{array}$} & $\begin{array}{c}\mu_{\text {surround }}\left(B, \mu_{n 1}\right) \\
\text { Figure } 8(\mathrm{a})\end{array}$ & \multicolumn{2}{|c||}{$\begin{array}{c}\mu_{\text {surround }}\left(B, \mu_{n 2}\right) \\
\text { Figure } 8(\mathrm{~b})\end{array}$} & \multicolumn{2}{|c||}{$\begin{array}{c}\mu_{\text {surround }}\left(B, \mu_{n 3}\right) \\
\text { Figure 8(c) }\end{array}$} & \multicolumn{2}{|c||}{$\begin{array}{c}\mu_{\text {surround }}\left(B, \mu_{n 4}\right) \\
\text { Figure 8(d) }\end{array}$} \\
\cline { 2 - 8 } & {$[N, \Pi]$} & $M$ & {$[N, \Pi]$} & $M$ & {$[N, \Pi]$} & $M$ & {$[N, \Pi]$} & $M$ \\
\hline \hline 1 (red) & {$[0.4,1]$} & 0.75 & {$[0.4,1]$} & 0.91 & {$[0.4,1]$} & 0.91 & {$[0.4,1]$} & 0.91 \\
2 (pink) & {$[0.29,0.83]$} & 0.54 & {$[0.5,0.90]$} & 0.72 & {$[0.5,1]$} & 0.94 & {$[0.5,1]$} & 0.95 \\
3 (orange) & {$[0,0]$} & 0.00 & {$[0,0]$} & 0.00 & {$[0,0.28]$} & 0.09 & {$[0.68,0.90]$} & 0.81 \\
4 (purple) & {$[0.6,1]$} & 0.98 & {$[0.6,1]$} & 0.98 & {$[0.6,1]$} & 0.98 & {$[0.6,1]$} & 0.98 \\
5 (green) & {$[0,0]$} & 0.00 & {$[0,0]$} & 0.00 & {$[0,0]$} & 0.00 & {$[0.82,0.89]$} & 0.86 \\
6 (yellow) & {$[0.37,1]$} & 0.78 & {$[0.7,1]$} & 0.94 & {$[0.7,1]$} & 0.96 & {$[0.7,1]$} & 0.96 \\
7 (blue) & {$[0.6,1]$} & 0.94 & {$[0.6,1]$} & 0.94 & {$[0.6,1]$} & 0.94 & {$[0.6,1]$} & 0.94 \\
8 (cyan) & {$[0.7,1]$} & 0.96 & {$[0.7,1]$} & 0.96 & {$[0.7,1]$} & 0.96 & {$[0.7,1]$} & 0.96 \\
\hline
\end{tabular}

Table 2: Results obtained for the target objects shown in Figure 6(c) with respect to the reference objects of Figure 6(b), using the membership function distance of Figure 7.

In this example we showed the influence of the notion of "near" introduced in the definition of Equation 16. We demonstrated the limitations of using very strict or loose functions. The examples in Figures $8(\mathrm{~b})$ and $8(\mathrm{c})$ show fuzzy landscapes which are in accordance with the situation. These two situations exhibit similar results, showing the flexibility in the choice of the function used to represent the notion of "near". Moreover, this function could be learned as in [16]. This would allow an automated use of this approach.

\section{Conclusion}

In this paper we have presented a fuzzy definition of the relation "surround". We first presented a definition that only considered the morphology of the reference objects. However, we saw that this definition is not adapted to evaluate the relation when the target objects are much smaller than the concavities and far from them. Therefore, the definition was adapted to take into account the shape of the reference object, as well as the size of the target objects and their distance to the concavities of the reference object. The proposed definition has nice geometric properties, it is in accordance with the intuition and is modeled as a fuzzy landscape which has shown interesting properties for image interpretation.

Results on real objects with complex shapes illustrate the interest of the proposed approach, and could then be further exploited for satellite image interpretation.

\section{References}

[1] I. Bloch. Fuzzy Relative Position between Objects in Image Processing: a Morphological Approach. IEEE Transactions on Pattern Analysis and Machine Intelligence, 21(7):657-664, 1999.

[2] K. Miyajima and A. Ralescu. Spatial organization in 2D segmented images: Representation and recognition of primitive spatial relations. Fuzzy Sets and Systems, 65:225-236, 1994.

[3] I. Bloch. Fuzzy spatial relationships for image processing and interpretation: a review. Image and Vision Computing, 23:89-110, 2005.
[4] I. Bloch, T. Géraud, and H. Maître. Representation and Fusion of Heterogeneous Fuzzy Information in the 3D Space for Model-Based Structural Recognition - Application to 3D Brain Imaging. Artificial Intelligence, 148(1-2):141-175, August 2003.

[5] I. Bloch. On fuzzy distances and their use in image processing under imprecision. Pattern Recognition, 32(11):1873-1895, 1999.

[6] A. Rosenfeld and R. Klette. Degree of adjacency or surroundedness. Pattern Recognition, 18(2):169$177,1985$.

[7] K. Miyajima and A. Ralescu. Spatial organization in $2 \mathrm{~d}$ images. In Third IEEE Conference on Fuzzy Systems, FUZZ-IEEE 1994, pages 100-105, 1994.

[8] P. Matsakis and S. Andréfouet. The fuzzy line between among and surround. In IEEE International Conference on Fuzzy Systems, FUZZ-IEEE 2002, volume 2, pages 1596-1601, 2002.

[9] Y. Mathet. Etude de l'expression en langue de l'espace et du déplacement : Analyse linguistique, modélisation cognitive et leur expérimentation informatique. PhD thesis, Université de CAEN, 2000.

[10] B. Bouchon-Meunier, M. Rifqi, and S. Bothorel. Towards general measures of comparison of objects. Fuzzy Sets and Systems, 84(2):143-153, 1996.

[11] D. Dubois, H. Prade, and C. Testemale. Weighted fuzzy pattern matching. Fuzzy Sets and Systems, 28(3):313-331, 1988.

[12] D. Hernandez, E. Clementini, and P. Di Felice. Qualitative distances. In A. Frank and W. Kuhn, editors, Spatial Information Theory - Proceedings of COSIT'95, pages 45-57, 1995.

[13] I. Bloch, O. Colliot, and R. Cesar. On the Ternary Spatial Relation Between. IEEE Transactions on Systems, Man, and Cybernetics SMC-B, 36(2):312327, April 2006.

[14] I. Bloch. Spatial reasoning under imprecision using fuzzy set theory, formal logics and mathematical morphology. International Journal of Approximate Reasoning, 41:77-95, 2006.

[15] C.B. Barber, D.P. Dobkin, and H. Huhdanpaa. The quickhull algorithm for convex hulls. ACM Transactions on Mathematical Software (TOMS), 22(4):469-483, 1996.

[16] O. Colliot, O. Camara, and I. Bloch. Integration of Fuzzy Spatial Relations in Deformable Models Application to Brain MRI Segmentation. Pattern Recognition, 39(8):1401-1414, August 2006. 